\title{
Ramucirumab in the second-line treatment of metastatic colorectal cancer: a narrative review of literature from clinical trials
}

\author{
Chou-Pin Chen ${ }^{1}$, Tao-Wei Ke ${ }^{2}$, Rebecca Cheng ${ }^{3}$, Jaw-Yuan Wang ${ }^{4,5,6,7}$ \\ ${ }^{1}$ Veterans General Hospital, Taichung; ${ }^{2}$ China Medical University Hospital, Taichung; ${ }^{3}$ Eli Lilly and Company, Taipei; ${ }^{4}$ Division of Colorectal \\ Surgery, Department of Surgery, Kaohsiung Medical University Hospital, ${ }^{5}$ Department of Surgery, Faculty of Medicine, College of Medicine, \\ ${ }^{6}$ Graduate Institute of Clinical Medicine, College of Medicine, ${ }^{7}$ Center for Cancer Research, Kaohsiung Medical University, Kaohsiung \\ Contributions: (I) Conception and design: CP Chen, TW Ke, R Cheng; (II) Administrative support: R Cheng; (III) Provision of study materials or \\ patients: None; (IV) Collection and assembly of data: CP Chen, TW Ke; (V) Data analysis and interpretation: R Cheng, JY Wang; (VI) Manuscript \\ writing: All authors; (VII) Final approval of manuscript: All authors. \\ Correspondence to: Prof. Jaw-Yuan Wang, MD, PhD. Division of Colorectal Surgery, Department of Surgery, Kaohsiung Medical University Hospital, \\ Kaohsiung Medical University, No. 100 Tzyou 1st Road, Kaohsiung. Email: Cy614112@ms14.hinet.net; jawyuanwang@gmail.com.
}

\begin{abstract}
The development of new anti-angiogenic agents targeting the vascular endothelial growth factor (VEGF) and epidermal growth factor receptor pathways has greatly expanded the therapeutic options for patients with metastatic colorectal cancer (mCRC). Although these new agents have considerably improved survival outcomes compared with conventional chemotherapeutic regimens, the optimal integration of these drugs into the management of patients with mCRC continues to develop. One particular challenge is the identification of patient subgroups that will benefit from treatment with a specific targeted agent. In RAISE, a phase III randomized, placebo-controlled clinical trial, the VEGF receptor 2 antagonist ramucirumab in combination with fluorouracil plus leucovorin and irinotecan demonstrated efficacy as a second-line treatment for patients with mCRC. Ramucirumab is approved for the treatment of patients with mCRC in Taiwan but, due to lack of reimbursement, is often reserved for use as a third-line or later treatment. This narrative review of prespecified and post-hoc analyses of the RAISE study data, and data from other ramucirumab trials and real-world studies, summarizes the efficacy and tolerability of ramucirumab in the second-line treatment of different subpopulations of patients with mCRC. The aim was to identify patients most likely to benefit from treatment with second-line ramucirumab, with a view to illustrating the potential benefit of integrating this regimen into Taiwanese or Asian treatment practice.
\end{abstract}

Keywords: Angiogenesis; colorectal cancer (CRC); narrative review; ramucirumab; VEGFR2 inhibition

Submitted Mar 09, 2020. Accepted for publication Aug 07, 2020.

doi: $10.21037 /$ tcr-20-608

View this article at: http://dx.doi.org/10.21037/tcr-20-608

\section{Introduction}

Worldwide, colorectal cancer (CRC) was the third most common cancer and the second leading cause of cancer death in 2018, with over 1.8 million new cases reported (1). The global distribution of CRC varies greatly across low to high human development index countries (2), but $~ 52 \%$ of the new cases of CRC reported in 2018 occurred in Asia (1). At diagnosis, $25 \%$ of patients have metastatic disease and nearly half of all patients without metastases will develop metastatic CRC (mCRC) (3).

In 2016, CRC accounted for $14.5 \%$ of all malignant tumors and for $12.0 \%$ of all cancer-related deaths in Taiwan. CRC was the most common cancer in men and the second most common cancer in women (4). Over 15,000 patients are diagnosed with CRC annually, with CRC accounting for $41.9 \%$ of all malignancies of the digestive organs and peritoneum in $2016(4,5)$. In the same year, 5,722 patients died from a colorectal or anal malignancy, and these tumor types were rated as the third and fourth most common 
causes of cancer-related death in men and women, respectively $(4,5)$.

Goals of treatment for unresectable mCRC include prolonging survival, reducing the impact of tumorrelated symptoms and maintaining quality of life (3). Fluoropyrimidines (e.g., capecitabine and fluorouracil) are the cornerstone of conventional palliative chemotherapy and are used in various combinations (3). Common combinations used for first-line therapy in patients with $\mathrm{mCRC}$ are fluorouracil plus leucovorin in combination with oxaliplatin (FOLFOX) or irinotecan (FOLFIRI) (3).

The development of new targeted agents has expanded the therapeutic options available for patients with mCRC (5). Both anti-angiogenic drugs targeting the vascular endothelial growth factor (VEGF) pathway and anti-epidermal growth factor receptor (EGFR) monoclonal antibodies have demonstrated antitumor activity when used in combination with conventional chemotherapeutic regimens $(6,7)$.

In line with global clinical guidelines (3), FOLFOX/ FOLFIRI and FOLFIRI/FOLFOX can be used interchangeably as first-/second-line therapies in patients with $\mathrm{mCRC}$ in Taiwan. Since 2010, the anti-VEGF monoclonal antibody bevacizumab has been reimbursed for the first-line treatment of $\mathrm{mCRC}$ in combination with FOLFIRI or fluorouracil/leucovorin (for a maximal cycle of 36 weeks) (8). Most patients with mCRC in Taiwan, especially those with a $R A S$ mutation, are treated with bevacizumab plus FOLFIRI in the first-line setting, with the exception of patients in whom irinotecan is contraindicated.

Several novel targeted agents are also available in the second-line setting. Ramucirumab, a human immunoglobulin G1 (IgG1) monoclonal antibody antagonist of VEGF receptor (VEGFR)-2, demonstrated efficacy in combination with FOLFIRI in the pivotal RAISE trial in patients whose $\mathrm{mCRC}$ progressed after bevacizumab plus FOLFOX (7) and has been approved in Taiwan accordingly. The Pan-Asian-adapted European Society for Medical Oncology consensus guidelines recommend ramucirumab plus FOLFIRI as a second-line therapy for the management of patients with mCRC (after first-line oxaliplatin) (9). However, in Taiwan (and other Asian countries such as China and Malaysia), ramucirumab plus FOLFIRI is more commonly used as a third-line or later treatment instead of in the recommended setting, due to lack of reimbursement (10).

Several factors, including patient and tumor characteristics, affect the efficacy of anti-angiogenic drugs and their suitability in certain patient populations (11-15). In this narrative review, we give an overview of overall survival (OS), progression-free survival (PFS) (see Figures 1 and 2, respectively), and tolerability data for ramucirumab in important patient subgroups using data from RAISE and subsequent exploratory studies, and other recent clinical trials and real-world evidence (RWE). Our aim is to summarize the clinical trial literature of second-line ramucirumab in clinically relevant patient subgroups, with a view to illustrating the potential benefit of integrating this regimen into Taiwanese or Asian treatment practice. We present the following article in accordance with the Narrative Review reporting checklist (available at http:// dx.doi.org/10.21037/tcr-20-608).

\section{Methods}

To identify exploratory and post-hoc analyses of the RAISE clinical trial, a literature search was conducted on MEDLINE (PubMed) using the following search terms "ramucirumab" and "colorectal cancer" and "RAISE". Only studies investigating the efficacy and safety of ramucirumab in the RAISE study population were included. Another search was conducted to identify other clinical trials, retrospective studies, and case studies not related to the RAISE study using the search terms "ramucirumab" and "colorectal cancer". No publication date range was set. Review articles, clinical trials in other advanced cancers, and studies of ramucirumab as a first-, fourth-, or higher-line of treatment were excluded. Both searches were conducted on July 1, 2020.

\section{Discussion}

\section{RAISE study}

In the multicenter, randomized phase III clinical trial, RAISE, ramucirumab plus FOLFIRI was compared with placebo plus FOLFIRI as a second-line treatment for mCRC following first-line FOLFOX-bevacizumab (7). A significant increase in OS was observed in patients in the ramucirumab arm compared with those in the placebo arm [hazard ratio (HR) 0.84, $\mathrm{P}=0.0219$ ] (7). Patients treated with ramucirumab/FOLFIRI also demonstrated a significant improvement in PFS vs. those treated with placebo/ FOLFIRI ( $\mathrm{P}=0.0005)(7)$. 


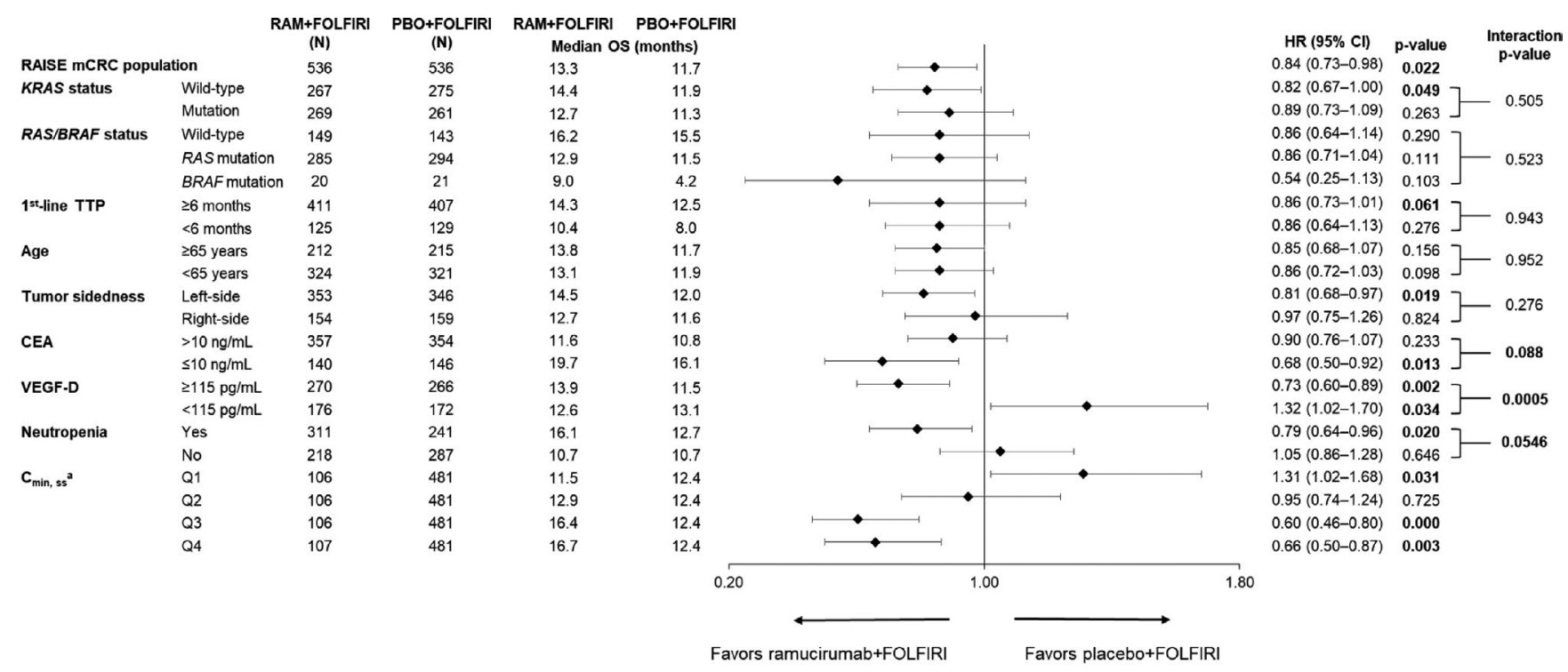

Figure 1 Forest plot showing overall survival in the general RAISE mCRC population and in patient subgroups. HRs and 95\% CIs are shown for subgroups as defined by baseline patient and tumor characteristics $(7,12,13,16-19)$. ', patients were grouped into four ramucirumab $\mathrm{C}_{\text {min,ss }}$ exposure quartiles: $<25 \%(\mathrm{Q} 1), 25 \%$ to $<50 \%(\mathrm{Q} 2), 50 \%$ to $<75 \%(\mathrm{Q} 3)$, and $\geq 75 \%$ (Q4). Potential interactions between treatmentgroup and subgroup variables were significant at a two-sided $\alpha$ level of 0.10 . CEA, carcinoembryonic antigen; CI, confidence interval; $\mathrm{C}_{\text {min,ss, }}$ minimum steady-state plasma concentration; FOLFIRI, fluorouracil/leucovorin/oxaliplatin; HR, hazard ratio; mCRC, metastatic colorectal cancer; OS, overall survival; PBO, placebo; RAM, ramucirumab; TTP, time to tumor progression; VEGF-D, vascular endothelial growth factor-D.

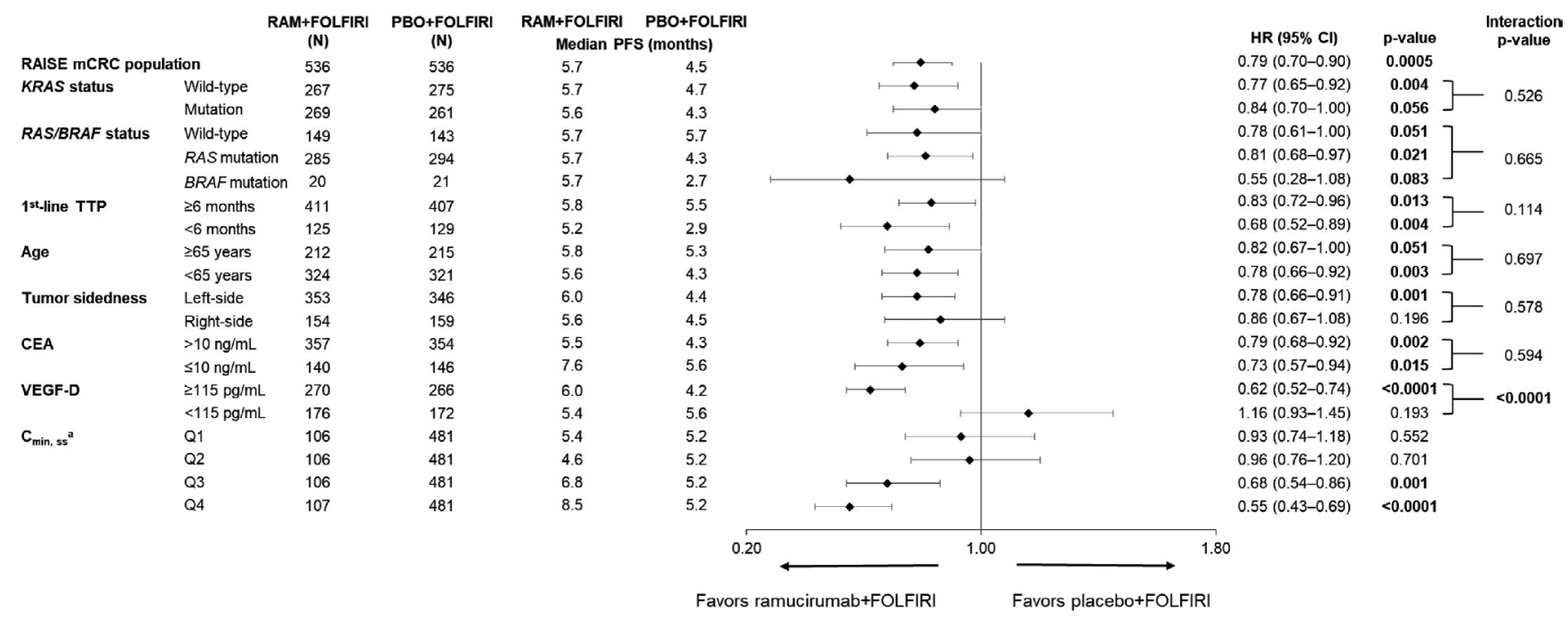

Figure 2 Forest plot showing progression-free survival in the general RAISE mCRC population and in patient subgroups. HRs and 95\% CIs are shown for subgroups as defined by baseline patient and tumor characteristics $(7,12,13,16-18)$. ${ }^{a}$, patients were grouped into four ramucirumab $\mathrm{C}_{\text {min,ss }}$ exposure quartiles: $<25 \%(\mathrm{Q} 1), 25 \%$ to $<50 \%(\mathrm{Q} 2), 50 \%$ to $<75 \%(\mathrm{Q} 3)$, and $\geq 75 \%$ (Q4). Potential interactions between treatment-group and subgroup variables were significant at a two-sided $\alpha$ level of 0.10 . CEA, carcinoembryonic antigen; CI, confidence interval; $\mathrm{C}_{\text {min,ss }}$, minimum steady-state plasma concentration; FOLFIRI, fluorouracil/leucovorin/oxaliplatin; HR, hazard ratio; mCRC, metastatic colorectal cancer; PBO, placebo; PFS, progression-free survival; RAM, ramucirumab; TTP, time to tumor progression; VEGF-D, vascular endothelial growth factor-D. 


\section{RAS/BRAF mutation status}

The presence of KRAS mutations have been identified as predictors of resistance to anti-EGFR therapy in patients with $\mathrm{mCRC}(12,20,21)$.

KRAS exon 2 mutation status was a stratification factor in RAISE; mutant KRAS tumors were present in approximately half of the patient population (12). In wildtype patients, treatment with ramucirumab/FOLFIRI significantly improved $\mathrm{OS}(\mathrm{P}=0.049)$ and $\mathrm{PFS}(\mathrm{P}=0.004)$ compared with placebo/FOLFIRI $(7,12)$. These findings were irrespective of post-discontinuation anti-EGFR therapy (12). For patients with mutant KRAS tumors, directional improvements in OS and PFS were seen in the ramucirumab arm $v s$. the placebo arm (12). Ramucirumab was effective $v s$. placebo regardless of KRAS mutation status, with no significant interaction between treatment effect and KRAS mutation status for either OS (interaction $\mathrm{P}=0.505$ ) or $\mathrm{PFS}$ (interaction $\mathrm{P}=0.526$ ) (12).

Recent evidence suggests that other RAS mutations (KRAS exon 3 and 4, NRAS) and BRAF mutations may also reduce the benefit of anti-EGFR therapies $(3,21)$. RAS/ $B R A F$ mutation information was available for $85 \%$ of the total RAISE population (13). Directional improvements in OS were observed in the ramucirumab arm in all patients compared with the placebo arm (13). Directional improvements for ramucirumab vs. placebo were also observed for PFS in patients with the BRAF mutation. A significant improvement in PFS was seen with ramucirumab over placebo in the $R A S$ mutation subgroup $(\mathrm{P}=0.021)$ (13). Treatment-by-mutation status interaction tests indicated that treatment effect did not differ significantly among the three mutation status subgroups for either survival outcome (OS interaction $\mathrm{P}=0.523$; PFS interaction $\mathrm{P}=0.665$ ) (13).

\section{Time to tumor progression (TTP) during first-line therapy}

In some cases, the aggressive nature of a disease has been known to impact treatment efficacy adversely, and rapid disease progression can act as a prognostic factor for survival, which may be useful when determining the nature of second-line or later therapy (22). Disease aggression can often be assessed using TTP during first-line therapy (12). Some clinical trials exclude patients with aggressive disease progression; in RAISE, however, patients with TTP $<6$ or $\geq 6$ months after the initiation of first-line treatment were analysed as prespecified subgroups (12). TTP on first-line therapy was a prognostic factor for patients on second-line therapy (Wald's $\mathrm{P}<0.0001)(12)$. No significant differences in OS and PFS were observed between those with TTP $<6$ months and those with TTP $\geq 6$ months (OS interaction $\mathrm{P}=0.943$; PFS interaction $\mathrm{P}=0.114$ ) (12).

\section{Advanced age}

As with any cancer treatment approach, the risk-benefit of a drug must be carefully considered in elderly patients who may be particularly vulnerable to treatment-emergent adverse events (TEAEs) $(12,23)$. In a post-hoc analysis of RAISE study data, ramucirumab/FOLFIRI demonstrated similar trends in OS improvement vs. placebo/FOLFIRI in both patients aged $<65$ and those aged $\geq 65$ years (12). An improvement in PFS vs. placebo was observed with ramucirumab in both older $(\mathrm{P}=0.051)$ and younger patients $(\mathrm{P}=0.003)$. However, treatment effect did not differ between the two subgroups (interaction $\mathrm{P}=0.952$ and $\mathrm{P}=0.697$, respectively) (12).

\section{Tumor sidedness}

Primary tumor location (i.e., left or right colon) has been linked to differences in clinical and biological characteristics between patients and may possibly be predictive of treatment response, particularly for anti-EGFR therapies $(14,15)$.

Tumor sidedness was known in $94.4 \%$ of the RAISE intention-to-treat population (13). Ramucirumab exhibited a trend for greater efficacy in patients with left-sided CRC than in those with right-sided disease; however, non-significant interaction tests meant that sidedness was not associated with the OS and PFS benefits of ramucirumab therapy (OS interaction $\mathrm{P}=0.276$; $\mathrm{PFS}$ interaction $\mathrm{P}=0.578$ ) (13).

\section{Baseline carcinoembryonic antigen (CEA) levels}

CEA, a glycoprotein produced by the gastrointestinal tract that can induce tumor angiogenesis, has been seen to increase proportionally with disease progression and may correlate inversely with OS and PFS in patients undergoing first-line therapy (24-26).

In the RAISE study, baseline CEA data were available for $93 \%$ of patients (median baseline CEA $34.1 \mathrm{ng} / \mathrm{mL}$ ). A prespecified subanalysis indicated that baseline CEA ( $<200 v s . \geq 200 \mu \mathrm{g} / \mathrm{L}$ ) did not affect the benefit obtained with second-line ramucirumab plus FOLFIRI (6). In a post-hoc study investigating a CEA cutoff of $10 \mathrm{ng} / \mathrm{mL}$ 
(based on $2 \times$ the upper limit of normal), 29\% of patients had CEA levels $\leq 10 \mathrm{ng} / \mathrm{mL}$ at baseline. Ramucirumab/ FOLFIRI demonstrated a relatively greater magnitude of improvement in OS and PFS in patients with baseline CEA levels $\leq 10 \mathrm{mg} / \mathrm{mL}$ than in those with CEA levels $>10 \mathrm{ng} / \mathrm{mL}$ (16). A significant interaction between CEA level and ramucirumab treatment effect was observed for OS at the 0.1 level (interaction $\mathrm{P}=0.088$ ) but not for PFS (interaction $\mathrm{P}=0.594$ ); therefore, a prospectively planned study to confirm the predictive role of baseline CEA is needed.

\section{Baseline VEGF-D levels}

Another prespecified endpoint of the RAISE study was to identify potential predictive biomarkers for the efficacy of ramucirumab (17). Using RAISE study data, Tabernero et al. (17) investigated a number of angiogenesis-related mediators as possible predictive factors through an adaptive signature design. Analyses focused on VEGF family members and their receptors, including VEGF-C, VEGF-D, soluble VEGFR-1, VEGFR-2, VEGFR-3 (plasma), and vascular VEGFR-2 (17).

Based on the findings of an exploratory subset analysis, in which only VEGF-D showed a strong association with treatment effect, patients were separated into high and low VEGF-D subgroups (<115 vs. $\geq 115 \mathrm{pg} / \mathrm{mL})$ (17). In the analyses, involving over $80 \%$ of the total RAISE population, high VEGF-D levels were seen in $61 \%$ of patients (17). In this high VEGF-D group, median OS $(\mathrm{P}=0.002)$ and PFS $(\mathrm{P}<0.0001)$ were significantly improved in the ramucirumab arm compared with the placebo arm, respectively (17). In contrast, for the low VEGF-D group, improvements in median OS and PFS were in favor of placebo/FOLFIRI (16). Significant differences in the OS and PFS benefits of ramucirumab were apparent between patients with high and low levels of VEGF-D (interaction $\mathrm{P}=0.0005$ and $\mathrm{P}<0.0001$, respectively) (17). Significantly worse survival outcomes were observed in patients in the high VEGF-D group receiving placebo/FOLFIRI (OS $\mathrm{P}=0.003$ ) (17). However, these findings were obtained using an assay that was developed for exploratory research purposes. Without a validated assay, this potential predictive factor cannot be utilized for clinical decision making.

\section{Exposure-response relationship of ramucirumab}

There is a close relationship between the pharmacokinetics of a drug and its efficacy. For many antibody-based anticancer therapies, increasing concentration correlates with an increase in effect (known as the "exposureresponse" phenomenon) (27-29). The exposure-response relationship of ramucirumab has been assessed in cancers such as gastric and non-small-cell lung cancer, and findings have indicated a positive relationship between exposure and survival outcomes $(30,31)$.

An exploratory investigation of RAISE data assessed the minimum steady-state plasma concentration $\left(\mathrm{C}_{\mathrm{min}, \mathrm{ss}}\right)$ and grouped patients into four ramucirumab $\mathrm{C}_{\text {min,ss }}$ exposure quartiles: $<25 \%$ (Q1), $25 \%$ to $<50 \%(\mathrm{Q} 2), 50 \%$ to $<75 \%$ (Q3), and $\geq 75 \%$ (Q4) (18). Approximately $85 \%$ of the total RAISE population was included in this analysis. A significant positive association was seen between increasing $\mathrm{C}_{\min , \mathrm{s}}$ and improved efficacy outcomes with ramucirumab (interaction $\mathrm{P}<0.0001$ for both OS and PFS). This association remained significant after adjustment for factors associated with OS or PFS (18). The two highest ramucirumab exposure groups (Q3 and Q4) demonstrated significantly higher OS than did placebo ( $\mathrm{P}=0.0003$ and $\mathrm{P}=0.003$, respectively) (18). Similar results were seen for PFS, with significant improvements seen in $\mathrm{Q} 3(\mathrm{P}=0.0014)$ and $\mathrm{Q} 4(\mathrm{P}<0.0001)$ but not in $\mathrm{Q} 1$ and Q2 (18).

\section{Tolerability of ramucirumab}

Ramucirumab has demonstrated acceptable tolerability across numerous clinical trials in other cancers $(30,31)$. In RAISE, most TEAEs were manageable with supportive care or dose modification. Almost one-third (29\%) of patients in the ramucirumab arm and $13 \%$ of patients in the placebo arm discontinued at least one treatment component (commonly FOLFIRI; only $4 \%$ of patients discontinued ramucirumab and $1 \%$ discontinued placebo). The incidence of grade $\geq 3$ TEAEs was higher in the ramucirumab arm than in the placebo arm (79\% vs. 62\%). The most commonly occurring grade $\geq 3$ TEAE was neutropenia (7). In general, the incidence of TEAEs across different patient subgroups were in line with observations from $\operatorname{RAISE}(7,12,17,18)$.

As advanced age is often associated with a higher frequency of TEAEs, age-subgroup analyses of RAISE data included not only patients aged $<65$ and $\geq 65$ years but also an age cutoff of $\geq 75$ years (12). The incidence of TEAEs was generally well-balanced between older ( $\geq 75$ years) and younger ( $<75$ years) patients. As expected, TEAEs associated with age (e.g., decreased appetite and fatigue) occurred more frequently in older patients; however, 
incidence was similar across treatment arms. The incidence of TEAEs of special interest was not increased in patients aged $\geq 65 v s$. $\geq 75$ years (12).

In subgroup analyses based on VEGF-D levels (<115 vs. $\geq 115 \mathrm{pg} / \mathrm{mL}$ ), the incidence of TEAEs in both subgroups was similar to that in the overall population from RAISE, although grade $\geq 3$ neutropenia was more frequent in the high VEGF-D group than in the low VEGF-D group ( $42 \%$ vs. $32 \%$, respectively) $(7,17)$.

A post-boc analysis of RAISE data explored the relationship between OS and treatment-emergent neutropenia (19). Across both treatment arms, rates of neutropenia increased in patients with certain baseline characteristics, including first-line TTP ( $\geq 6$ months), race (Asian), and sex (female). Median time to the first neutropenic event was 2.6 weeks ( 3.0 weeks for grade $\geq 3$ ) in the ramucirumab arm and 3.5 weeks in the placebo arm (3.9 weeks for grade $\geq 3$ ) (19). In the ramucirumab arm, median OS was significantly longer in patients with neutropenia than in those without neutropenia (16.1 vs. 10.7 months; $\mathrm{P}<0.0001)$; corresponding data for the placebo arm were 12.7 vs. 10.7 months $(\mathrm{P}=0.007)(19)$. Ramucirumab demonstrated significantly improved OS over placebo in patients with any-grade neutropenia $(\mathrm{P}=0.020)$ but not in patients without neutropenia. An interaction between treatment effect and the occurrence of neutropenia was observed for OS (interaction $\mathrm{P}=0.055$ ). Ramucirumab/ FOLFIRI demonstrated similar efficacy in patients with grade $\geq 2$ or $\geq 3$ neutropenia (19).

The relationship between the early incidence of TEAEs and survival outcomes has been explored in a post-hoc analysis of RAISE (32). OS and PFS in patients reporting TEAEs within the first 42 days of receiving ramucirumab/ FOLFIRI were assessed. In line with the above-mentioned study (19), neutropenia was associated with improved OS [grade $\leq 2$ : HR $0.67,95 \%$ confidence interval (CI): 0.490.91 ; grade $\geq 3$ : HR $0.82,95 \%$ CI: $0.64-1.05 ; \mathrm{P}=0.022$ ] but not PFS (grade $\leq 2$ : HR $0.85,95 \%$ CI: $0.66-1.10$, grade $\geq 3$ : HR 1.03, 95\% CI: 0.83-1.28; P=0.379). Interestingly, diarrhea was associated with both worse OS (grade $\leq 2$ : HR 0.96, 95\% CI: 0.76-1.20; grade $\geq 3$ : HR 2.72, 95\% CI: 1.67-4.44; $\mathrm{P}=0.001$ ) and PFS (grade $\leq 2$ : HR $1.02,95 \%$ CI: $0.83-1.23$; grade $\geq 3$ : HR 2.22, $95 \%$ CI: $1.43-3.45$; $\mathrm{P}=0.005)$. Similar associations between neutropenia and diarrhea, and survival outcomes were not apparent in the placebo/FOLFIRI group (32).

\section{Other clinical studies}

Efficacy data from other recent clinical and real-world studies of second-line treatment ramucirumab in patients with $\mathrm{mCRC}$ are summarized in Table 1.

Phase II studies have demonstrated the efficacy of ramucirumab in the second-line therapy of patients with CRC. In one study conducted in 102 patients with KRAS selected wild-type CRC previously treated with FOLFOX or capecitabine/oxaliplatin plus bevacizumab, the addition of ramucirumab to a combination of irinotecan and cetuximab improved PFS significantly vs. the combination alone (33). In another study of 153 patients with mCRC who had progressed on irinotecan-based therapy, median PFS in patients who had received ramucirumab plus modified FOLFOX-6 (mFOLFOX-6) was comparable to that seen in those treated with FOLFOX-6 alone (HR 1.116, 95\% CI: 0.713-1.745; P=0.0623) (34).

\section{RWE}

Very little RWE for the efficacy of ramucirumab in the second-line treatment of patients with mCRC was identified. In one retrospective study of 43 patients with mCRC across six health centers in Japan, ramucirumab/ FOLFIRI as second- or later-line therapy demonstrated a positive impact on survival outcomes, with the median duration of PFS on second-line ramucirumab being equivalent to that seen in RAISE (5.4 months). Patients receiving second-line therapy showed prolonged median OS and PFS compared with patients receiving third- or later-line ramucirumab (35). Another retrospective Japanese study investigated the impact of second-line ramucirumab/ FOLFIRI on survival outcomes in 74 patients with mCRC (36). Overall, median PFS and OS were 6.2 and 17.0 months, respectively, findings in line with those from RAISE. Median PFS was somewhat lower in patients who received first-line bevacizumab (5.0 vs. 8.0 months in those not receiving first-line bevacizumab), but this difference did not reach significance (36). Finally, in two case studies from Japan, treatment with ramucirumab/mFOLFOX-6 following first-line treatment with panitumumab/FOLFIRI was beneficial in both patients (both men aged $\geq 65$ years) with mCRC. Tumor shrinkage was noted in one patient (37).

\section{Summary and conclusions}

This narrative review of evidence from the large RAISE 
Table 1 Summary of survival data from phase II clinical trials and from real-world studies investigating the use of ramucirumab as a second- or later-line treatment in patients with mCRC (33-37)

\begin{tabular}{|c|c|c|c|c|c|}
\hline Reference & Study design & Treatment arms & $\mathrm{N}$ & Patient population & Findings \\
\hline \multicolumn{6}{|l|}{ Phase II evidence } \\
\hline $\begin{array}{l}\text { Hochster et al. } \\
\text { [2018] (33) }\end{array}$ & $\begin{array}{l}\text { A randomized phase II } \\
\text { trial of } \mathrm{IC} \pm \text { ramucirumab } \\
\text { as } 2 \text { nd-line treatment in } \\
\text { patients with } \mathrm{mCRC}\end{array}$ & IC vs. mICR & 102 & $\begin{array}{l}\text { KRAS selected } \\
\text { wild-type patients } \\
\text { with advanced and } \\
\text { measurable CRC }\end{array}$ & $\begin{array}{l}\text { Median PFS: Overall: } 5.8 \text { months } \\
\text { PFS HR: mICR vs. IC: } 0.65 ; \mathrm{P}=0.068\end{array}$ \\
\hline \multicolumn{6}{|c|}{ Real world evidence } \\
\hline $\begin{array}{l}\text { Yoshihiro et al. } \\
\text { [2019] (35) }\end{array}$ & $\begin{array}{l}\text { A retrospective analysis of } \\
\text { patients with } \mathrm{mCRC} \text { who } \\
\text { received ramucirumab } \\
\text { plus FOLFIRI at any } \\
\text { therapy line at six } \\
\text { healthcare institutions in } \\
\text { Japan }\end{array}$ & $\begin{array}{l}\text { Ramucirumab plus } \\
\text { FOLFIRI (2nd- vs. } \\
\geq 3 \text { rd-line therapy and } \\
\text { high vs. low RDI) }\end{array}$ & 43 & $\begin{array}{l}\text { Patients with } \\
\text { mCRC }\end{array}$ & $\begin{array}{l}\text { Median PFS: all patients: } 4.8 \text { months; } \\
\text { 2nd-line therapy: } 5.4 \text { months; } \geq 3 \text { rd- } \\
\text { line therapy: } 2.8 \text { months; high RDI: } \\
\text { 3.2 months; low RDI: } 5.7 \text { months } \\
\text { (HR =2.7; P=0.004) } \\
\text { Median OS: all patients: } 17.3 \text { months; } \\
\text { 2nd-line therapy: } 17.4 \text { months; } \geq 3 \text { rd- } \\
\text { line therapy: } 13.0 \text { months; high RDI: } \\
18.5 \text { months; low RDI: } 14.4 \text { months }\end{array}$ \\
\hline $\begin{array}{l}\text { Hagino et al. } \\
\text { [2017] (37) }\end{array}$ & $\begin{array}{l}\text { Two case studies of } \\
\text { patients with mCRC who } \\
\text { received ramucirumab } \\
\text { plus FOLFIRI as a 2nd- } \\
\text { line treatment }\end{array}$ & $\begin{array}{l}\text { Ramucirumab plus } \\
\text { FOLFIRI }\end{array}$ & 2 & $\begin{array}{l}\text { Older ( } \geq 65 \text { years) } \\
\text { male patients with } \\
\text { mCRC }\end{array}$ & $\begin{array}{l}\text { Treatment with ramucirumab was } \\
\text { beneficial and was administered for } \\
\geq 7 \text { months in both patients. Tumor } \\
\text { shrinkage was observed in one } \\
\text { patient }\end{array}$ \\
\hline
\end{tabular}

CRC, colorectal cancer; FOLFIRI, fluorouracil/leucovorin/oxaliplatin; FOLFOX, fluorouracil plus leucovorin in combination with oxaliplatin; $\mathrm{HR}$, hazard ratio; IC, irinotecan and cetuximab; mCRC, metastatic colorectal cancer; mFOLFOX, modified FOLFOX; mICR, IC with ramucirumab; N, number of patients; OS, overall survival; PFS, progression-free survival; RDI, relative dose intensity. 
phase III clinical trial (7), its exploratory studies $(12,16,18)$, as well as data from other clinical trials $(33,34)$ and RWE (35-37) has shown that ramucirumab has consistent efficacy across multiple patient subpopulations and can prolong patient survival when administered as part of second-line therapy for mCRC. Although the results of exploratory and retrospective studies (conducted in non-randomized populations) should be treated with caution, those reviewed here provide important information that can be used to help identify patient subgroups that will potentially benefit from treatment with ramucirumab, and to indicate areas for further research.

Recently, reimbursement guidelines in Taiwan have been revised, effective at the beginning of 2019 (38), to allow bevacizumab to be combined with either irinotecan (FOLFIRI)- or oxaliplatin (FOLFOX)-based chemotherapy. A recent observational study from Taiwan reported PFS to be higher in patients who had received second-line treatment with bevacizumab/aflibercept plus FOLFOX-6 vs. FOLFOX-6 alone (39). The reimbursement of other anti-angiogenic drugs on the basis of demonstrated efficacy increases the possibility of using ramucirumab with FOLFIRI in the second-line setting. However, lack of reimbursement for ramucirumab strongly limits its use in clinical practice in Taiwan and other Asian countries.

\section{Acknowledgments}

The authors would like to thank Dr. Elinor Wylde and Gill Gummer (Rx Communications, Mold, UK) for medical writing assistance with the preparation of this article, funded by Eli Lilly and Company.

Funding: This work was funded by Eli Lilly and Company. One of the authors, involved in the conception of this review, is an employee of Eli Lilly and Company.

\section{Footnote}

Reporting Checklist: The authors have completed the Narrative Review reporting checklist. Available at http:// dx.doi.org/10.21037/tcr-20-608

Peer Review File: Available at http://dx.doi.org/10.21037/tcr20-608

Conflicts of Interest: All authors have completed the ICMJE uniform disclosure form (available at http://dx.doi. org/10.21037/tcr-20-608). Dr. Cheng reports personal fees from Eli Lilly and Company, during the conduct of the study. Dr. Cheng is an employee of Eli Lilly and Company. The other authors have no conflicts of interest to declare.

Etbical Statement: The authors are accountable for all aspects of the work in ensuring that questions related to the accuracy or integrity of any part of the work are appropriately investigated and resolved.

Open Access Statement: This is an Open Access article distributed in accordance with the Creative Commons Attribution-NonCommercial-NoDerivs 4.0 International License (CC BY-NC-ND 4.0), which permits the noncommercial replication and distribution of the article with the strict proviso that no changes or edits are made and the original work is properly cited (including links to both the formal publication through the relevant DOI and the license). See: https://creativecommons.org/licenses/by-nc-nd/4.0/.

\section{References}

1. The Global Cancer Observatory. Colorectal cancer fact sheet 2018. Available online: http://gco.iarc.fr/today/data/ factsheets/cancers/10_8_9-Colorectum-fact-sheet.pdf (Accessed 24 December 2018).

2. Arnold M, Sierra MS, Laversanne M, et al. Global patterns and trends in colorectal cancer incidence and mortality. Gut 2017;66:683-91.

3. Van Cutsem E, Cervantes A, Nordlinger B, Arnold D; ESMO Guidelines Working Group. Metastatic colorectal cancer: ESMO Clinical Practice Guidelines for diagnosis, treatment and follow-up. Ann Oncol 2014;25 Suppl 3:iii1-9. Erratum in: Ann Oncol 2015;26 Suppl 5:v174-7.

4. Health Promotion Administration, Ministry of Health and Welfare. Cancer Registry Annual Report 2016, Taiwan. Available online: https://www.hpa.gov.tw/Pages/Detail. aspx? nodeid=269\&pid=10227 (Accessed 18 January 2019).

5. Health Statistics. Ministry of Health and Welfare. Statistics of General Health and Welfare 2018, Taiwan. Available online: https://www.mohw.gov.tw/lp-4614-2. html (Accessed 16 December 2019).

6. Foubert F, Matysiak-Budnik T, Touchefeu Y. Options for metastatic colorectal cancer beyond the second line of treatment. Dig Liver Dis 2014;46:105-12.

7. Tabernero J, Yoshino T, Cohn AL, et al. RAISE Study Investigators. Ramucirumab vs. placebo in combination with second-line FOLFIRI in patients with metastatic colorectal carcinoma that progressed during or after 
first-line therapy with bevacizumab, oxaliplatin, and a fluoropyrimidine (RAISE): a randomised, double-blind, multicentre, phase 3 study. Lancet Oncol 2015;16:499-508.

8. National Health Insurance, Drug Payment Regulations Amendment Checklist, 2017, Taiwan. Available online: https://www.nhi.gov.tw/Resource/ bulletin/6917_1060053987-1.pdf(Accessed 7 February 2019).

9. Yoshino T, Arnold D, Taniguchi H, et al. Pan-Asian adapted ESMO consensus guidelines for the management of patients with metastatic colorectal cancer: a JSMOESMO initiative endorsed by CSCO, KACO, MOS, SSO and TOS. Ann Oncol 2018;29:44-70.

10. Taiwan Food and Drug Administration, Ministry of Health and Welfare. 2015. Available online: https://www.fda.gov. tw/MLMS/H0001D.aspx? Type=Lic\&LicId=60000999 (Accessed 7 February 2019).

11. De Stefano A, Carlomagno C. Beyond KRAS: Predictive factors of the efficacy of anti-EGFR monoclonal antibodies in the treatment of metastatic colorectal cancer. World J Gastroenterol 2014;20:9732-43.

12. Obermannová R, Van Cutsem E, Yoshino T, et al. Subgroup analysis in RAISE: a randomized, double-blind phase III study of irinotecan, folinic acid, and 5-fluorouracil (FOLFIRI) plus ramucirumab or placebo in patients with metastatic colorectal carcinoma progression. Ann Oncol 2016;27:2082-90.

13. Yoshino T, Portnoy DC, Obermannová R, et al. Biomarker analysis beyond angiogenesis: RAS/RAF mutation status, tumour sidedness, and second-line ramucirumab efficacy in patients with metastatic colorectal carcinoma from RAISE-a global phase III study. Ann Oncol 2019;30:124-31.

14. Lee GH, Malietzis G, Askari A, et al. Is right-side colon cancer different to left-side colorectal cancer? A systematic review. Eur J Surg Oncol 2015;41:300-8.

15. Schrag D, Weng S, Brooks G, et al. The relationship between primary tumor sidedness and prognosis in colorectal cancer. J Clin Oncol 2016;34:abstr 3505.

16. Yoshino T, Obermannová R, Bodoky G, et al. Baseline carcinoembryonic antigen as a predictive factor of ramucirumab efficacy in RAISE, a second-line metastatic colorectal carcinoma phase III trial. Eur J Cancer 2017;78:61-9.

17. Tabernero J, Hozak RR, Yoshino T, et al. Analysis of angiogenesis biomarkers for ramucirumab efficacy in patients with metastatic colorectal cancer from RAISE, a global, randomized, double-blind, phase III study. Ann Oncol 2018;29:602-9.

18. Cohn AL, Yoshino T, Heinemann V, et al. Exposure- response relationship of ramucirumab in patients with advanced second-line colorectal cancer: exploratory analysis of the RAISE trial. Cancer Chemother Pharmacol 2017;80:599-608.

19. Grothey A, Yoshino T, Bodoky G, et al. Association of baseline absolute neutrophil counts and survival in patients with metastatic colorectal cancer treated with secondline antiangiogenic therapies: exploratory analyses of the RAISE trial and validation in an electronic medical record data set. ESMO Open 2018;3:e000347.

20. Perincheri S, Hui P. KRAS mutation testing in clinical practice. Expert Rev Mol Diagn 2015;15:375-84.

21. Yen LC, Uen YH, Wu DC, et al. Activating KRAS mutations and overexpression of epidermal growth factor receptor as independent predictors in metastatic colorectal cancer patients treated with cetuximab. Ann Surg 2010;251:254-60.

22. Shitara K, Matsuo K, Yokota T, et al. Prognostic factors for metastatic colorectal cancer patients undergoing irinotecan-based second-line chemotherapy. Gastrointest Cancer Res 2011;4:168-72.

23. Kuboki Y, Mizunuma N, Ozaka M, et al. Grade 3/4 neutropenia is a limiting factor in second-line FOLFIRI following FOLFOX4 failure in elderly patients with metastatic colorectal cancer. Oncol Lett 2011;2:493-8.

24. Fakih MG, Padmanabhan A. CEA monitoring in colorectal cancer. What you should know. Oncology (Williston Park) 2006;20:579-87; discussion 588, 594, 596 passim.

25. Prager GW, Braemswig KH, Martel A, et al. Baseline carcinoembryonic antigen (CEA) serum levels predict bevacizumab-based treatment response in metastatic colorectal cancer. Cancer Sci 2014;105:996-1001.

26. Michl M, Stintzing S, Fischer von Weikersthal L, et al. FIRE-3 Study Group. CEA response is associated with tumor response and survival in patients with KRAS exon 2 wild-type and extended RAS wild-type metastatic colorectal cancer receiving first-line FOLFIRI plus cetuximab or bevacizumab (FIRE-3 trial). Ann Oncol 2016;27:1565-72.

27. Feng Y, Roy A, Masson E, et al. Exposure-response relationships of the efficacy and safety of ipilimumab in patients with advanced melanoma. Clin Cancer Res 2013;19:3977-86.

28. Wang J, Song P, Schrieber S, et al. Exposure-response relationship of T-DM1: insight into dose optimization for patients with HER2-positive metastatic breast cancer. Clin Pharmacol Ther 2014;95:558-64.

29. Zhu M, Tang R, Doshi S, et al. Exposure-response analysis 
of rilotumumab in gastric cancer: the role of tumour MET expression. Br J Cancer 2015;112:429-37.

30. Tabernero J, Ohtsu A, Muro K, et al. Exposure-response analyses of ramucirumab from two randomized, phase III trials of second-line treatment for advanced gastric or gastroesophageal junction cancer. Mol Cancer Ther 2017;16:2215-22.

31. Smit EF, Garon EB, Reck M, et al. Exposure-response relationship for ramucirumab from the randomized, double-blind, phase 3 REVEL trial (docetaxel vs. docetaxel plus ramucirumab) in second-line treatment of metastatic non-small cell lung cancer. Cancer Chemother Pharmacol 2018;82:77-86.

32. Lim HH, Hopkins AM, Rowland A, et al. Effect of early adverse events on survival outcomes of patients with metastatic colorectal cancer treated with ramucirumab. Target Oncol 2019;14:743-8

33. Hochster H, Catalano P, O'Dwyer P, et al. Randomized trial of irinotecan and cetuximab (IC) vs. irinotecan, cetuximab and ramucirumab (ICR) as 2nd line therapy of advanced colorectal cancer (CRC) following oxaliplatin and bevacizumb based therapy: Result of E7208. J Clin Oncol 2018;36:abstr 3504.

34. Moore M, Gill S, Asmis T, et al. Randomized phase II study of modified FOLFOX-6 in combination with ramucirumab or icrucumab as second-line therapy in

Cite this article as: Chen CP, Ke TW, Cheng R, Wang JY. Ramucirumab in the second-line treatment of metastatic colorectal cancer: a narrative review of literature from clinical trials. Transl Cancer Res 2020;9(9):5645-5654. doi: 10.21037/tcr20-608 patients with metastatic colorectal cancer after disease progression on first-line irinotecan-based therapy. Ann Oncol 2016;27:2216-24.

35. Yoshihiro T, Kusaba H, Makiyama A, et al. Efficacy and safety of ramucirumab plus modified FOLFIRI for metastatic colorectal cancer. Int J Clin Oncol 2019;24:508-15.

36. Suzuki T, Shinozaki E, Osumi H, et al. Second-line FOLFIRI plus ramucirumab with or without prior bevacizumab for patients with metastatic colorectal cancer. Cancer Chemother Pharmacol 2019;84:307-13.

37. Hagino S, Miyata T, Sakamoto K. Experience of ramucirumab plus FOLFIRI as second-line treatment for metastatic colorectal cancer in our hospital. Gan To Kagaku Ryoho 2017;44:1559-61.

38. Health Agency Announcement: Amendment of Payment Rules for Bevacizumab-containing Drugs 2019. Available online: http://www.tsim.org.tw/helth/hel328_m710.html (Accessed 16 December 2019).

39. Tsai HL, Huang CW, Ma CJ, et al. An observational study of vascular endothelial growth factor inhibitors as secondline treatment for metastatic colorectal cancer treated with bevacizumab plus FOLFIRI beyond progression: the association with RAS mutation and tumor sidedness. Transl Cancer Res 2019;8:2357-70. 